\title{
A new mutation in the human pres gene and its effect on prestin function
}

\author{
TIMEA TOTH ${ }^{1}$, LEVENTE DEAK $^{2,4}$, FERENC FAZAKAS $^{3}$, JING ZHENG $^{4}$, \\ LASZLO MUSZBEK $^{3}$ and ISTVAN SZIKLAI ${ }^{1}$
}

\author{
${ }^{1}$ Medical and Health Science Center, Department of Otolaryngology, University of Debrecen, Debrecen; \\ ${ }^{2}$ Department of Otolaryngology, St. Imre Hospital, Budapest; ${ }^{3}$ Medical and Health Science Center, Clinical Research Center, \\ Haemostasis, Thrombosis and Vascular Biology Research Group of the Hungarian Academy of Sciences, \\ University of Debrecen, Debrecen, Hungary; ${ }^{4}$ Auditory Physiology Laboratory, Departments of Communication \\ Sciences and Disorders, The Neuroscience Institute, Northwestern University, Evanston, IL, USA
}

Received March 28, 2007; Accepted May 21, 2007

\begin{abstract}
The electromotility of cochlear outer hair cells (OHCs) is a major factor in cochlear amplification that enhances the sensitivity of hearing in humans. Prestin is associated with presumed conformational changes in an integral membrane protein. Prestin knockout (-/-) mice display loss of $\mathrm{OHC}$ electromotility and a $40-$ to $60-\mathrm{dB}$ reduction in cochlear sensitivity in vivo. In the present study we described the results of a direct sequencing mutation in the pres gene that was found in genetic screening performed in 47 patients characterized by non-syndromic, mild-to-moderate hearing impairment (30-70 dB) and in 50 control subjects from Hungary, after exclusion of $G J B(G J B 2, G J B 6)$ mutations in the background. Only one patient and his normal-hearing father showed a heterozygous missense mutation (R150Q/ WT) in the 6th coding exon of the pres gene. None of the 50 control subjects with normal hearing carried this mutation. Electrophysiological studies on the R150Q (homozygous and heterozygous) prestin mutant transiently transfected into reporting cells demonstrated nonlinear capacitance functions (NLC) as a signature of OHC electromotility. The capacitance function in human kidney cell line TSA 201 was similar for wild-type prestin and the mutant. However, for the mutant the voltage where the maximal charge displacement occurred $\left(V_{1 / 2}\right)$ significantly shifted in the hyperpolarizing direction $(\sim 15 \mathrm{mV})$. This is the first genetic and electrophysiological analysis of a human mutation in a coding exon of the pres gene by 47 patients with non-syndromic, sensorineural, mildto-moderate hearing impairment; although the pathogenic role of the R150Q mutation is not unambiguous.
\end{abstract}

Correspondence to: Dr Timea Toth, Medical and Health Science Center, Department of Otolaryngology, University of Debrecen, 4012 Debrecen, Nagyerdei krt. 98, Hungary

E-mail: ttimi@dote.hu

Key words: prestin, pres, hearing impairment, R150Q mutation, transfected cells, patch-clamp

\section{Introduction}

Mammalian peripheral auditory processing exhibits sharp tuning and low threshold due to a unique sensory cell type, the outer hair cell (OHC). OHCs are not only receptor cells which convert the mechanical energy of sound into electrical signals (receptor potential), but they are capable of transforming electrical signals into mechanical activity. This is the so-called fast-, or electromotility (1-3). Electromotility of OHCs is a frequency-following shortening and elongation in the frequency range of human hearing which feeds energy back to the cochlear partition on a cycle-by-cycle basis. This feedback mechanism is considered to be the cochlear amplifier. Electromotility of OHCs improves human hearing by $\sim 50 \mathrm{~dB}$, to the sensitivity threshold level of the inner hair cells which are the real receptor cells for hearing and which activate the cochlear neurons. The very fast motor capability of the OHCs is due to a modified transporter molecule, prestin, that undergoes conformational changes upon depolarizing and hyperpolarizing alterations of the OHC's membrane potential (4). Concerted action of the prestin molecules in the lateral $\mathrm{OHC}$ membrane manifests in cell shortening and elongation on a $\mu \mathrm{sec}$ to msec time scale.

Prestin expression has been observed in OHCs as well as in vestibular hair cells (VHCs), however not in the lateral plasma membrane or in the stereociliary membrane of VHCs (5). Analysis of its amino acid sequence indicates that it is a hydrophobic membrane protein that belongs to the SLC26A anion-transport family (6). Ten mammalian members of the SLC26A family have been identified (SLC26A1-10). Prestin is an $81-\mathrm{kDa}$ protein consisting of 746 amino acids with similarities to pendrin and other members of the solute carrier sulphate-anion transporter family (4). It has also been determined that both the $\mathrm{N}$-terminus and the $\mathrm{C}$-terminus of prestin are located on the intracellular side, and prestin has a highly hydrophobic core with 12 transmembrane domains (7). Intracellular anions $\mathrm{Cl}^{-}$and $\mathrm{HCO}_{3}^{-}$may act as voltage sensors of prestin, or are required for prestin's function $(8,9)$. In contrast to the highly conserved hydrophobic core, the $\mathrm{C}$ terminus of prestin is the least conserved region of the protein, because it has only $25-35 \%$ homology with its other 
SLC26A relatives (7). Prestin first appeared to behave as an incomplete anion transporter (10), but recent theoretical study suggests a transport function as well (11).

Three human genes of the SLC26A family (SLC26A2, $S L C 26 A 3$, and SLC26A4) are known to be associated with different human recessive hereditary diseases: distrophic dysplasia, congenital chloride diarrhea and Pendred syndrome (12). Different mutations in SLC26A4 are responsible for either syndromic hearing loss (Pendred syndrome) or nonsyndromic recessive hearing impairment (13).

The human gene SLC26A5 (pres), encoding prestin, contains 21 exons and is localized on the long arm of chromosome 7 (7q22.1) and chromosome 5 for the human and mouse isoforms (14). This gene is alternately spliced to create four isoforms of prestin with variable lengths of the Cterminus (15). Prestin knockout (-/-) mice display loss of $\mathrm{OHC}$ electromotility and a 40- to $60-\mathrm{dB}$ reduction in cochlear sensitivity in vivo (16), despite apparently intact mechanotransduction (17). Prestin knockout (-/-) mice also lose frequency selectivity (17). Wu and colleagues reported that prestin knockout (-/-) mice display significant hearing impairment at high frequencies before cell death occurs. Shorter OHCs in prestin knockout (-/-) mice suggest that prestin also plays a structural role in the lateral wall of the $\mathrm{OHC}$. The structural instability of the $\mathrm{OHC}$ in prestin knockout (-/-) mice may be a cause of the degeneration of these OHCs. In addition, transmission electron microscopy showed no obvious abnormality in the stereocilia, lateral wall, tight junctions and synapses of the prestin knockout (-/-) mouse OHCs (18).

The role and the extent of prestin gene defects in human non-syndromic hearing impairment (HI) are poorly understood. A single nucleotide change in the 2nd intron of SLC26A5 has been reported to be associated with hearing loss (15). This IVS2-2A $>$ G DNA sequence variation occurs in the first coding exon 3 splice acceptor site of the pres gene. It has been suggested that this mutation leads to aberrant mRNA splicing and results in non-syndromic moderate-to-profound sensorineural $\mathrm{HI}$ predominantly at high frequencies in patients with the heterozygous IVS2-2A>G mutation, and severe-toprofound HI by homozygous probands. However, further study demonstrated that the SLC26A5 IVS2-2A $>$ G variant may not occur more frequently in hearing impaired patients than in controls, and heterozygosity for this transition may not be sufficient to cause hearing loss (19). Cheatham and colleagues demonstrated that measurements of nonlinear capacitance in isolated $\mathrm{OHCs}$, as well as the voltage dependence and sensitivity of motor function, did not differ in the heterozygote knockout and wild-type mice, and neither did auditory threshold and frequency selectivity. These results suggest that one copy of the pres gene is able to compensate for the deleted copy and that heterozygous mice do not suffer hearing impairment (20).

The purpose of this study was to examine whether hearing was impaired as a result of a certain mutation in the SLC26A5 gene in humans. We describe the results of a direct sequencing mutation analysis in the pres gene that was performed in 47 patients characterized with mild and moderate hearing impairment (30-60 dB) and in 50 control subjects from Hungary. All individuals were tested for the most common genetic defects for hearing impairment in Hungary as well, the connexin 26 and connexin 30 mutations (21). When a genetic defect was detected, the pathogenic role of the given mutation was examined through a functional study.

\section{Materials and methods}

All 47 subjects participating in the study were from Hungary. The age of the patients varied between 3 and 30 years (mean, 15.6 years). No consanguinity was found among the patients. The hearing impairment was mild-to-moderate (30-70 dB), non-syndromic, sensorineural and bilateral in all cases. Information on the patients' medical history was obtained in personal interviews with the affected individuals or with their unaffected relatives. Patients who had any obvious risk factors (syphilis, rubeola, toxoplasma, CMV, HIV, meningitis, ototoxic drug use, head and acoustic trauma, premature birth, fetal alcohol syndrome, thyroid disease, kidney disease, neurodegenerative disorders, or other congenital and chromosomal abnormalities) were not included in this study. Informed consent was obtained from each individual and from the parents of children. This study was approved by the Ethics Committee of the University of Debrecen.

Audiologic methods. All persons involved in the study underwent otoscopic and audiometric examinations by using age-appropriate methods. The pure-tone threshold audiometric configuration was classified according to the guidelines of the European Work Group on Genetics of Hearing Impairment (22). Asymmetry of the hearing threshold ( $>10-\mathrm{dB}$ hearing level difference between the ears at least at two frequencies) was not observed for any subject. Distortion oto-acoustic emission (sound pressure level is plotted against frequency) showed no response on either side in all the patients tested. Auditory brainstem response (ABR) measurements were performed in selected cases. Auditory steadystate response (ASSR - to modulated $0.5-\mathrm{kHz}$ and $4-\mathrm{kHz}$ tones) was measured in only one genetically affected case.

Genetic analysis. Genetic analysis of GJB2 (connexin 26, NM_004004.3), GJB6 (connexin 30, AL590096.16) and pres (ENSG00000170615) genes was based on gene structure information provided by their GenBank accession nos. and/or Ensemble gene ID's.

Genomic DNA was extracted from 'buffy coat' by using QIAamp DNA blood mini kit (Qiagen) according to the manufacturer's instructions. For PCR amplification and DNA sequencing of the GJB2 and pres genes, custom primer pairs were designed using Primer Premier 5 (Premier Biosoft International). The primer pairs amplified the coding exons and the surrounding intronal and/or UTR regions of the respective genes (Table I). PCR conditions were as follows: $100 \mathrm{ng}$ genomic DNA was amplified in a $50-\mu 1$ volume containing $5 \mu \mathrm{l}$ of Buffer I, $0.4 \mu \mathrm{l}$ of $25 \mathrm{mM}$ dNTPs, 2 units of AmpliTaq Gold ${ }^{\circledR}$ DNA polymerase (Applied Biosystems), 20 pmole of each specific primer and 5\% DMSO. PCR reactions were run on a GeneAmp 9600 (Applied Biosystems) PCR instrument according to the following conditions: after an initial denaturation/polymerase activation at $96^{\circ} \mathrm{C}$ for $10 \mathrm{~min}, 35$ cycles were performed (1-min denaturation at 
Table I. The primer pairs used to amplify the coding exons of the pres gene and the surrounding intronal and/or UTR regions of the GJB2 and GJB6 genes.

Forward primer $\left(5^{\prime}-3^{\prime}\right)$

TATATTTGCGGGGATCGAC AGCCACTGTGATAGCAGTAGC

TCAGAAGAACTTGGAACACC

TCCACCCACCTTGAAAAT

AGATGATGTGCAGGACTGG

CCATATCAGATACTCTGAACC

CCCGTGTCTACTAAAAATACA

GGCTGGTGTGAATTAGTGC

GAACATTCATCTCTAAAGGTGC

CAACCAGAGCAAATAAGTATCC

ATGAAGAGATAGGCTGTAGTGC

AACCAACTTATGCCCTGC

GCTTCAAAGCTACCATCG

AGATCTCCCCTTTTTCTGG

CAAATGCACCAGCAAACC

GCAGCAGCCTTCCATCTCC

GATAATCTGACCCAGAAAGC

GAACACAAGAGCAGATTTTCC

GTCTTCTATCACGAAAAACA

CTGATATCCAGAGTGTGG

GACCTCACTCGGAATAGATT

Exon 21

GJB2

Exon 2

Seq 1

Seq2

Seq3

Seq4

GJB6

Exon 2

Seq 1

Seq2

Seq3

Seq4

Seq5

Seq6

\section{GCATTCGTCTTTTCCAGAGC}

GAAGAGGAAGTTCATCAAG

ACCAGCATTGGAAAGATCTG

GCATATTTGCTTCCTTCCAGC

AGTCAGAACTCAGAACACTGC

GACAACGCAGGCAAGAAGC

ATCGTACTCACCATCTGTGAGC
Reverse primer (5'-3')

TTAGGGAAAAAGCGCGAC ACTACCTCTGACCCACTCTCC CAGCATTCATTTTTCTCTCC CAATTGTTTGAGGACAGCA TGACCATGCTATTTCTTTGG CCACCGTGTAGTAAGACC GGGAATAAAGAGAAAAGTACC TTTTGGCAGAATTGAGAGC ATTAGCCAGGTGTGGTAGC TTCACCATGTTAGCCAGG CCCTACACAGCTCACTGG TTGGCCCTTTCTCATACC ACACACACATGCTACTTTTACC AGTCAACATTGTCATCTCTGC GCTCAGACTTTTCACTTTGACC CAACCCACAAATCCCATCCTC ATAACTGACCTTATCAGGTGC TTGTTGAAAAGAGAGCCTAGC ATATTAAGCTTCACCCCA GTACAATACATCTTGCTAGG TCGATGCTTAGCTTACAAAG

CCTACAGGGGTTTCAAATGG

ATTCACTCTTTATCTCCCC

ATGACCCGGAAGAAGATGCTG

CCTATACCCGGCTAGACAGC
AGAGGAACTCAATGATGAGC TCAGGTTGGGTGCTGAAGC TCAGAGTGGGGTCACTCAGC $96^{\circ} \mathrm{C}, 1-\mathrm{min}$ annealing at an exon-specific temperature and 1 -min elongation at $72^{\circ} \mathrm{C}$ ) followed by a 10 -min incubation at $72^{\circ} \mathrm{C}$. The annealing temperature was $57^{\circ} \mathrm{C}$ for GJB2, $54^{\circ} \mathrm{C}$ for Prestin exons $2-21$ and $62^{\circ} \mathrm{C}$ for pres exon 1 . The PCR products were checked by $3 \%$ agarose gel electrophoresis and purified using High Pure 96 UF Cleanup kit (Roche). The sequencing primers in the case of pres were the same as those used for the PCR reactions. For GJB2, besides the PCR primers, additional sequencing primers were also used.

Sequencing was carried out in $20-\mu 1$ reaction volumes in a 96-well GeneAmp 9600 PCR machine using the BigDye Terminator v3.1 Cycle Sequencing kit (Applied Biosystems) containing 3.2 pmole sequencing primer, $8 \mu$ I PCR template, $4 \mu 1$ dye terminators, and $4 \mu 15 \mathrm{X}$ sequencing buffer. Reactions were cycled by running at $96^{\circ} \mathrm{C}$ for one min, followed by 25 cycles of $96^{\circ} \mathrm{C}$ for $30 \mathrm{sec}, 50^{\circ} \mathrm{C}$ for $15 \mathrm{sec}$ and $60^{\circ} \mathrm{C}$ for $4 \mathrm{~min}$. Unincorporated dye terminators and residual salts were removed by using the DyeEx 96 kit (Qiagen). Samples were run on 3100 Avant DNA analyzer (Applied Biosystems). The sequencing results were aligned against reference sequences using Seqscape software v.2 (Applied Biosystems).

GJB6 analysis. The del(GJB6-D13S1830) mutation of the GJB6 (connexin 30) was evaluated by allele-specific PCR method. Using primers for 1 (gccatgcatgtggcctacta), rev1 (actatctgaaatcagctcattc) and for 2 ,del (cattgttgtgaactaacctcca, GenBank no. AL590096.16) a 441-bp PCR product was generated for wild-type alleles and a 480-bp fragment was produced for an allele representing the deletion. 


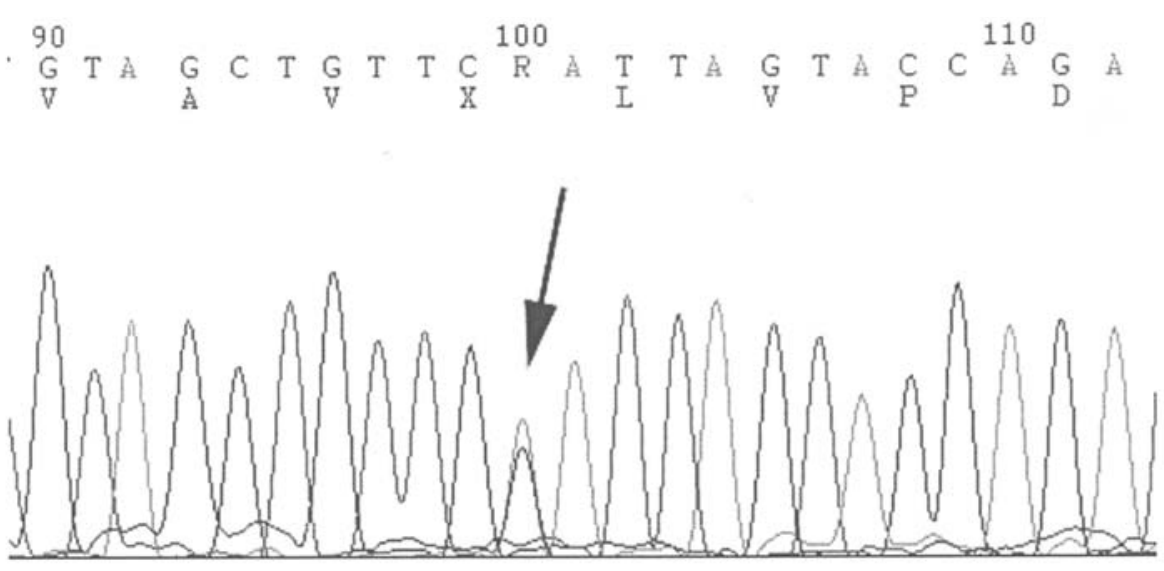

Figure 1. DNA sequencing of coding exon 6 of the pres gene. R150Q heterozygous missense mutation showing G $\rightarrow \mathrm{A}$ transversion in codon 150 by patient Pr-H1.

Pres DNA constructs and generation of mutant forms of prestin. Gerbil Prestin ( $g$ Prestin) was cloned into the vector pcDNA3.1 (4). A QuickChange ${ }^{\circledR}$ Site-Directed Mutagenesis kit (Stratagene, La Jolla, CA, USA) was used to generate wild-type (wt) prestin and the R150Q mutant. The nucleotide exchanges were confirmed by DNA sequencing as described previously (23). The heterozygote form was mimicked by mechanical mixing in the ratio of $1: 1$ of the wt and R150Q form. This procedure was used previously by Matsuda et al (24).

Cell culture and transfection. TSA201 cells were transiently transfected with the wild-type and the R150Q mutant prestin form. Both had a GFP tag on the N-terminal for the visualization of the transfected cells. The transfection reagent was Effectene (Qiagen, Valencia, CA) (7). cDNA (0.4 $\mu \mathrm{g})$ was used for one $35-\mathrm{mm}$ culture dish. The TSA cell culturing was as previously described (23). To perform whole cell recordings, the cells were dissociated $48 \mathrm{~h}$ after transfection with non-enzymatic Cell Dissociation solution (Sigma C5914) and transferred to a Poly-D-lysine-covered dish (Becton Dickinson).

Patch-clamp recording. The recording glass pipette (Borosilicate glass, Fisher, TW 150-4) tip resistance was between 1.5 and $2.4 \mathrm{M} \Omega$. The solution inside the pipette contained $130 \mathrm{nM} \mathrm{CsCl}, 2 \mathrm{nM} \mathrm{MgCl}{ }_{2}, 10 \mathrm{nM}$ EGTA, and $10 \mathrm{nM}$ HEPES at $\mathrm{pH}$ 7.2. The bath solution (ionic blocking solution) had the following composition: $99 \mathrm{nM} \mathrm{NaCl}, 20 \mathrm{nM}$ TEA-Cl, $2 \mathrm{nM} \mathrm{CoCl} 2,1.47 \mathrm{nM} \mathrm{MgCl} 2,1 \mathrm{nM} \mathrm{CaCl} 2,10 \mathrm{nM}$ HEPES and $4.6 \mathrm{~g}$ glucose at $\mathrm{pH}$ 7.2. Glucose was used to adjust the osmolarity to $300 \pm 4$ mosmol $1^{-1}$. The cell selection and recording procedure was similar to that described previously (23).

Data collection. The recorded capacitance functions were stored and fitted to the first derivative of a two-state Boltzmann function (25).

$$
\mathrm{C}_{v}=\mathrm{C}_{\text {lin }}+\frac{\mathrm{Q}_{\max }}{\alpha e^{\frac{V-V_{1 / 2}}{\alpha}}\left(1+e^{-\frac{V-V_{1 / 2}}{\alpha}}\right)^{2}}
$$

The maximal charge transfer $\left(\mathrm{Q}_{\max }\right)$ depends on cell size, consequently the $\mathrm{Q}_{\max }$ was divided by linear capacitance $\left(\mathrm{C}_{\text {lin }}\right)$ and named charge density (fC/pF) (26). The software Igor Pro (WaveMetrics, Lake Oswego, OR) was used for data analysis and curve fitting.

Statistics. To analyze the differences between electrical properties of mutant and wild-type pres, we transfected two dishes simultaneously (one with wild-type and another with the mutant). The cells were from the same passage. We first measured at least 12 cells from the wild-type group and afterward also $\sim 12$ cells from the mutant. Thereby two populations were compared. The statistical significance levels were estimated by unpaired two-tailed Student's ttests. All data were presented as average and standard deviation. A probability value of $\mathrm{p}<0.05$ was considered significant.

\section{Results}

Genetic analysis. All 47 subjects selected for this study suffered from a sporadic, sensorineural, bilateral, mild-tomoderate hearing disorder based on pure tone audiometry (at frequencies $0.5,1,2$, and $4 \mathrm{kHz}$ ), with unknown etiology and with normal middle ear function. None of the patients had a mutation in the coding region of GJB2. The 309-kb deletion $[\Delta(G J B 6-\mathrm{D} 13 \mathrm{~S} 1830)] G J B 6$ was not detectable in any samples as well. Each coding exon of the human pres was analysed in all cases. One p.R150Q heterozygous missense mutation was detected in exon 6 in one of the 47 patients ( $\mathrm{Pr}-\mathrm{H} 1)$. This mutation represents a $\mathrm{G} \rightarrow \mathrm{A}$ transversion in codon 150 , resulting in an amino acid change from arginine (basic) to glycine (polar) (Fig. 1). The degree of hearing impairment in this child with the p.R150Q heterozygous genotype was estimated at 60-70 dB at all frequencies by auditory steady state response (ASSR) measurement. Further pres sequencing analysis was performed on his parents to verify the inheritance of the R150Q mutation. It was shown that the 34-year-old father (Pr-H2) also had the mentioned DNA change in heterozygous form. Pure-tone threshold audiograms, otoacoustic emission, as well as ABR measurements showed normal patterns in both parents. The p.R150Q mutation 


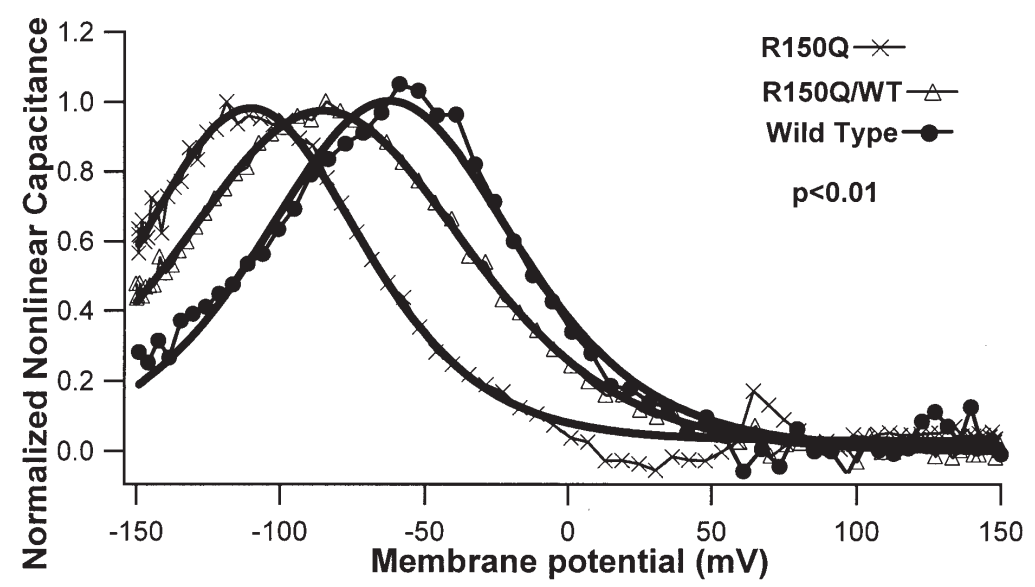

Figure 2. Normalized nonlinear capacitance functions of the R150Q mutant and wild-type prestin in transfected TSA cells. Each curve represents the individual cell that approximately describes the mean of the population. Thin lines with different markings represent the actual data, and thick lines show curve fitting according to equation 1 . In the case of the R150Q mutant form, the voltage where the maximal prestin movement occurred ( $\left.V_{1 / 2}\right)$, shifted to the hyperpolarizational direction $(\mathrm{p}<0.005)$ compared to wild-type prestin. For clearer presentation each NLC curve was normalized to 1 because their charge density did not show significant differences.

screening in the pres gene was performed on 50 Hungarian control subjects with reported normal hearing. None of them carried this mutation.

Nonlinear capacitance (NLC) of the R150Q mutant. Several transfected TSA cells were measured and the recorded values within the same genotype (wt or mutant) were averaged. The daily control was required because, depending on the transfection efficiency, the charge density can change from batch to batch. The three main electrical properties (charge density, $V_{1 / 2}$ and $\alpha$ ) of the voltage-dependent capacitance function were compared between the wild-type and the mutant.

The average charge density of the R150Q mutant was $9 \pm 6 \mathrm{fC} / \mathrm{pF}(\mathrm{n}=16)$. The corresponding control wild-type control displayed $7 \pm 4 \mathrm{fC} / \mathrm{pF}(\mathrm{n}=12)$. The difference between the two population means was not significant $(p>0.05)$. The recorded charge density for the hetero form (R150Q/WT) was $14 \pm 8 \mathrm{fC} / \mathrm{pF}(\mathrm{n}=13)$ and compared to its own daily control, $10 \pm 8 \mathrm{fC} / \mathrm{pF}(\mathrm{n}=14)$. The level of significance was $\mathrm{p}>0.05$.

The peak voltage of the capacitance function $\left(V_{1 / 2}\right)$ of the R150Q homozygote mutant was $-112 \pm 12 \mathrm{mV}$. The daily control of the wt prestin was $-63 \pm 7 \mathrm{mV}$. This $49-\mathrm{mV}$ hyperpolarization shift was significant $(\mathrm{p}<0.01)$. The heterozygote R150Q/WT mutant displayed $-89 \pm 17 \mathrm{mV}$ while the daily control was $-58 \pm 8 \mathrm{mV}$. The difference $(29 \mathrm{mV})$ was still significant $(\mathrm{p}<0.001)$. Thus, both homozygous and heterozygous forms of the R150Q mutation showed significant differences in their $V_{1 / 2}$ compared to the wild-type.

The $\alpha$ values, the slope factor of the NLC curves, were similar in each case; for the homozygote mutant it was $32 \pm 2 \mathrm{mV}$ (control value, $29 \pm 5 \mathrm{mV}$ ) and for the heterozygote mutant it was $29 \pm 5 \mathrm{mV}$ (control value, $28 \pm 4 \mathrm{mV}$ ) (Fig. 2). There was no statistically significant difference between the mutant and its own control $(\mathrm{p}>0.05)$.

\section{Discussion}

This is the first genetic and electrophysiological analysis of a human mutation in a coding exon of the pres gene in patients with non-syndromic, sensorineural, mild-to-moderate hearing impairment.

Examination of hearing impaired patients $(n=47)$, after exclusion of $G J B$ mutations in the background, resulted in the discovery of a heterozygous mutation in the pres gene. This mutation was also found in the father of the subject who was without hearing loss. No similar mutation was seen in the age- and gender-matched control group of normal hearing listeners $(n=50)$. Out of the tested individuals with a hearing impairment of $>40 \mathrm{~dB}$ and a sloping audiometric chart towards the high frequencies associated with the absence of evoked oto-acoustic emission (TOAE) and GJB mutations (connexin 26 and connexin 30 ) only 1 patient ( $\mathrm{Pr}-\mathrm{H} 1)$ showed a heterozygous missense mutation in the pres gene. The mutation was a R150Q/WT in the 6th coding exon. Although this young male carrier did not produce oto-acoustic emissions, his father (Pr-H2) with the same mutations did. This finding makes it unlikely that the mutant prestin was the causative factor in the hearing impairment.

Electrophysiological studies on an R150Q mutant prestin transfected kidney cell line exhibited a hyperpolarization shift in the voltage-dependent nonlinear peak capacitance of $\sim 30 \mathrm{mV}$. Since in a heterozygote subject, the normal gene copy can still produce wild-type prestin in the outer hair cells, it is unlikely that the mutation described here was responsible for the hearing loss. We can only speculate on the putative influence of such a mutation in the outer hair cell function in e.g. a homozygous genotype.

First, the exchange of Arg to Gly decreases the effectiveness of phosphorylation upon the prestin molecule. Consequently, mutant prestin is less modifiable by metabolic influences and hence its contribution in dynamic stiffness regulation of the $\mathrm{OHC}$ lateral wall decreases (27).

Second, Arg is a positively charged amino acid, which may closely be involved in the charge carrier function of prestin. As this is a basic mechanism in the cochlear amplifier role of the $\mathrm{OHCs}$ in mammalian hearing this mutation can change the charge carrier characteristics of the prestin molecules. 
Third, an 30-mV hyperpolarizing shift in the voltagedependent nonlinear peak capacitance in homozygotes can affect the transfer curve of electromotility (a voltage to movement conversion in the OHCs) with a consequence of energy-feedback change or feedback phase change resulting in hearing impairment.

In other words, mutant homozygous prestin performs smaller elongation displacements and greater contractions than the wild-type. Also, the electromotility magnitude of contraction + elongation decreases.

To summarize, it is at least possible that one copy of this mutation also produces a highly variable phenotype, maybe even normal hearing in some cases. Certainly in the animal model, the (+/-) genotype corresponds to normal function (for knockout mouse). Maybe in the case of modified prestin, the contributions of R150 and Q150 vary from case to case and so does prestin function and hearing loss.

\section{Acknowledgements}

This study was supported by the Hungarian National Research Fund (OTKA-K63743), by ETT 206/2006 and by the Medical and Health Science Center, University of Debrecen, Mecenatura grant (16/2005). The authors are grateful to all subjects for their participation in this study. We further thank Prof. Peter Dallos (Northwestern University, Evanston) for helpful discussions on the research project and manuscript, as well as Judit Szilvássy (our Department) and Laszlo Noszek (Department of ENT, University of Budapest) for their audiological work.

\section{References}

1. Brownell WE, Bader CR, Bertrand D and de Ribaupierre Y: Evoked mechanical responses of isolated cochlear outer hair cells. Science 227: 194-196, 1985.

2. Kachar B, Brownell WE, Altschuler R and Fex J: Electrokinetic shape changes of cochlear outer hair cells. Nature 322: 365-368, 1986.

3. Ashmore JF: A fast motile response in guinea-pig outer hair cells: the cellular basis of the cochlear amplifier. J Physiol 388: 323-347, 1987.

4. Zheng J, Long KB, Shen W, Madison LD and Dallos P: Prestin is the motor protein of cochlear outer hair cells. Nature 405: 149-155, 2000.

5. Adler HJ, Belyantseva IA, Merritt RC Jr, Frolenkov GI, Dougherty GW and Kachar B: Expression of prestin, a membrane motor protein, in the mammalian auditory and vestibular periphery. Hear Res 184: 27-40, 2003

6. Mount DB and Romero MF: The SLC26 gene family of multifunctional anion exchangers. Pflugers Arch 447: 710-721, 2004.

7. Zheng J, Long KB, Shen W, Madison LD and Dallos P: Prestin topology: localization of protein epitopes in relation to the plasma membrane. NeuroReport 12: 1929-1935, 2001.
8. Oliver D, He DZZ, Klöcker N, et al: Intracellular anions as the voltage sensor of prestin, the outer hair cell motor protein. Science 292: 2340-2343, 2001.

9. Rybalchenko V and Santos-Sacchi J: $\mathrm{Cl}^{-}$flux through a nonselective, stretch-sensitive conductance influences the outer hair cell motor of the guinea-pig. J Physiol 547: 873-891, 2003.

10. Dallos P and Fakler B: Prestin, a new type of motor protein. Nat Rev Mol Cell Biol 3: 104-111, 2002.

11. Muallem D and Ashmore J: An anion antiporter model of prestin, the outer hair cell motor protein. Biophys J 90: 4035-4045, 2006.

12. Everett LA and Green EDA: Family of mammalian anion transporters and their involvement in human genetic diseases. Hum Mol Genet 8: 1883-1891, 1999.

13. Li XC, Everett LA, Lalwani AK, et al: A mutation in PDS causes non-syndromic recessive deafness. Nat Genet 18: 215-217, 1998.

14. Zheng J, Long KB, Matsuda KB, Madison LD, Ryan AD and Dallos PD: Genomic characterization and expression of mouse prestin, the motor protein of outer hair cells. Mamm Genome 14: 87-96, 2003.

15. Liu XZ, Ouyang XM, Xia XJ, et al: Prestin, a cochlear motor protein, is defective in non-syndromic hearing loss. Hum Mol Genet 12: 1155-1162, 2003.

16. Liberman MC, Gao J, He DZZ, Wu X, Jia S and Zuo J: Prestin is required for electromotility of the outer hair cell and for the cochlear amplifier. Nature 419: 300-304, 2002.

17. Cheatham MA, Huynh KH, Gao J, Zuo J and Dallos P: Cochlear function in Prestin knockout mice. J Physiol 560: 821-830, 2004.

18. Wu X, Gao J, Guo Y and Zuo J: Hearing threshold elevation precedes hair cell loss in prestin knockout mice. Brain Res Mol Brain Res 126: 30-37, 2004.

19. Tang HY, Xia A, Oghalai JS, Pereira FA and Alfrod RL: High frequency of the IVS2-2A $>$ G DNA sequencing variation in SLC26A5, encoding the cochlear motor protein prestin, precludes its involvement in hereditary hearing loss. BMC Med Genet 6: 30, 2005.

20. Cheatham MA, Zheng J, Huynh KH, et al: Cochlear function in mice with only one copy of the prestin gene. J Physiol 569: 229-241, 2005.

21. Toth T, Kupka S, Haack B, et al: GJB2 mutations in patients with non-syndromic hearing loss from Northeastern Hungary. Hum Mutat 6: 631-632, 2004.

22. European Work Group on Genetics of Hearing Impairment. Info Letter, Nov. 2, 1996.

23. Deák L, Zheng J, Orem A, et al: Effects of cyclic nucleotides on the function of prestin. J Physiol 563: 483-496, 2005.

24. Matsuda K, Zheng J, Du GG, Klocker N, Madison LD and Dallos P: N-linked glycosylation sites of the motor protein prestin: effects on membrane targeting and electrophysiological function. J Neurochem 89: 928-938, 2004.

25. Santos-Sacchi J: Reversible inhibition of voltage-dependent outer hair cell motility and capacitance. J Neurosci 10: 3096-3110, 1991.

26. Oliver D and Fakler B: Expression density and functional characteristics of the outer hair cell motor protein are regulated during postnatal development in rat. J Physiol 519: 791-800, 1999.

27. He DZ, Jia S and Dallos P: Prestin and the dynamic stiffness of cochlear outer hair cells. J Neurosci 8: 9089-9096, 2003. 